\title{
Wernicke's Encephalopathy - 'Pushing the Envelope' of Patient's Profile: A Case Report
}

\author{
B. Jayaprakash ${ }^{a}$ Karthik N. Rao ${ }^{a}$ Navin Patil ${ }^{b}$ Dipanjan Bhattacharjee ${ }^{b}$ \\ Mohit Maden ${ }^{\text {b N.R. Rau }}{ }^{\mathrm{c}}$ \\ ${ }^{a}$ Department of Medicine, ${ }^{b}$ Department of Pharmacology, Kasturba Medical College, Manipal Campus, Manipal \\ University, and ${ }^{\mathrm{C}}$ Department of Medicine, Yenepoya Medical College, Mangalore, Karnataka, India
}

\section{Key Words}

Hepatitis A - Thiamine - Wernicke's encephalopathy ·

Korsakoff's psychosis

\begin{abstract}
Wernicke's encephalopathy (WE), an acute neuropsychiatric condition, is caused by thiamine deficiency. Traditionally, it has been associated with patients with a background of alcoholism. However, in the past few decades, with increasing trends in the incidence of WE among patients without a history of alcohol consumption, a pressing need was felt to examine the existing guidelines for the management of WE and its sequelae. The need for a revision was felt as the guidelines for the management of WE were developed around the premise that this affliction is observed mainly among alcoholics. In light of the opportunity presented to us by one of our patients who did not 'fit the bill' of a traditional case of WE, we decided to compare and contrast the management of WE among patients with and without a background of alcoholism. After analyzing the available data on WE among alcoholics and non-alcoholics, we concluded that a high degree of suspicion should be kept in mind if any of the classical features are observed, with a special emphasis on eliciting the underlying causes through a detailed history.
\end{abstract}

Furthermore, thiamine supplementation at a lower dose of 100-200 mg should be initiated first, followed by laboratory and radiological investigations.

(c) 2016 S. Karger AG, Basel

\section{Introduction}

Wernicke's encephalopathy (WE), first observed by a Polish neurologist Dr. Carl Wernicke in 1881, refers to an acute neuropsychiatric condition caused by the deficiency of thiamine [1]. Thiamine or vitamin B1 occupies a central position in cerebral energy utilisation by virtue of being an integral part of various metabolic pathways [2]. Deficiency of thiamine along with its associated errors of metabolism, especially in conditions of increased metabolic demands, is believed to lead to an initially reversible neuronal injury due to the production of increased reactive oxygen species and damage to the blood-brain barrier [3]. Clinically, ataxia of gait, oculomotor dysfunction, and encephalopathy are its hallmark presentations.

WE has been associated with chronic alcoholism. However, in recent years, various cases of WE have been observed in patients without a history of alcohol consumption. A variety of underlying conditions, for in-

\section{KARGER}

E-Mail karger@karger.com

www.karger.com/aon
(C) 2016 S. Karger AG, Basel

0972-7531/16/0233-0188\$39.50/0 


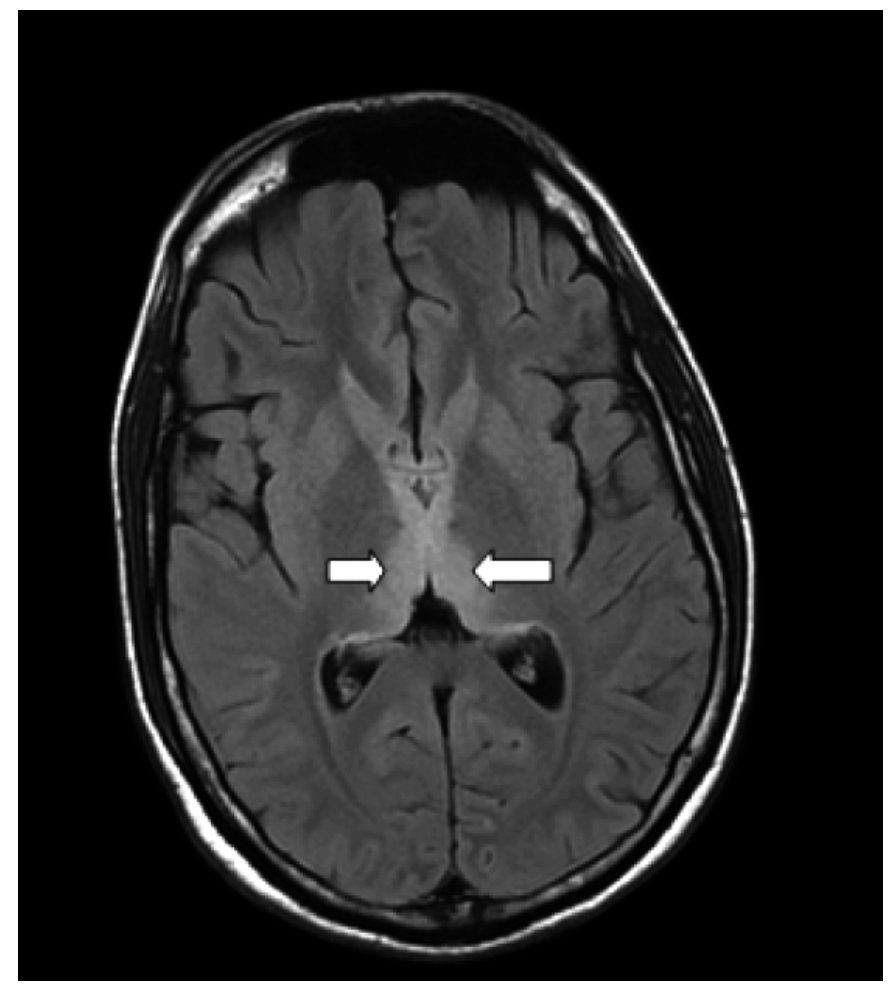

Fig. 1. MRI brain (axial) showing hyper-intensities in the medial thalamus region demarcated by arrows.

stance, Hodgkin's lymphoma [4], vomiting [5], celiac disease [6], infectious diseases [7], and post-gastrectomy status $[4,8]$ have been identified as potential risk factors for the development of WE. In our clinical practice, we encountered one such patient, who was diagnosed as a case of non-alcoholic WE with an underlying history of prolonged vomiting following hepatitis A infection. The purpose of reporting this unique case is to contribute to the expanding literature highlighting the wide spectrum of clinical settings in which non-alcoholic WE may ensue. Furthermore, this also prompts us to revisit the present management guidelines for WE, especially among nonalcoholic WE patients.

\section{Case Report}

A 23-year-old man presented at the hospital with altered sensorium and slurring of speech of one-day duration. A detailed history revealed that the patient was suffering from vomiting and jaundice since the past 1 month. On probing further, it was determined that the patient did not suffer from any previous co-morbidity nor did he have any history of alcohol consumption. No additional symptomatology was noted during the time of presentation.

WE - 'Pushing the Envelope' of Patient's Profile

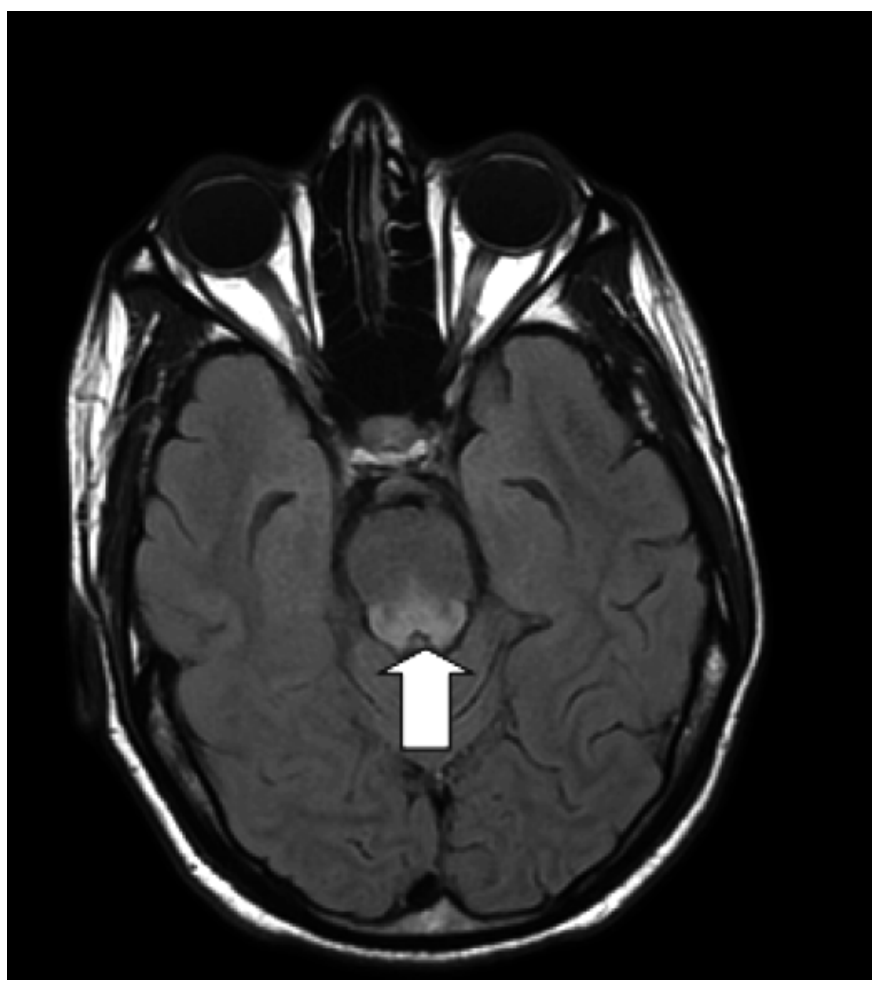

Fig. 2. MRI brain (axial) showing hyper-intensities in the inferior colliculus and periaqueductal region demarcated by arrow.

General examination revealed the patient to be hemodynamically stable, with an anomaly of deep icterus. And the cardiopulmonary evaluation did not reveal any abnormalities. However, an analysis of the neurological state of the patient revealed mental sluggishness, disorientation, and anterograde amnesia. Neurological signs of ophthalmoplegia, nystagmus, and ataxia were also observed.

In light of presenting symptoms and examination findings, a strong suspicion of WE developed. Besides WE, other possibilities, for instance, hepatic encephalopathy, cerebrovascular accident were also considered.

Further, keeping the patient's debilitated state in mind, he was immediately administered intravenous thiamine $100 \mathrm{mg}$ sixth hourly for 5 days; thereafter he was administered oral thiamine 100 mg once daily for 1 week.

Laboratory investigations were carried out to determine the underlying cause for the patient's symptoms. Most of the hematological and biochemical parameters were found to be within normal limits, with the exception of elevated bilirubin and a deranged liver profile, confirming the development of jaundice. Pre- and post-supplementation thiamine levels were not measured as it is not a routine investigation. Furthermore, the utility of thiamine levels in the management of a case of WE was suspected. MRI brain imaging revealed symmetrical T2W and FLAIR hyper-intensities involving bilateral medial thalami (fig. 1), periaqueductal region and colliculi (fig. 2), caudate nucleus (fig. 3), and mammillary bodies (fig. 4) that were consistent with WE. Furthermore, serological

Ann Neurosci 2016;23:188-193 


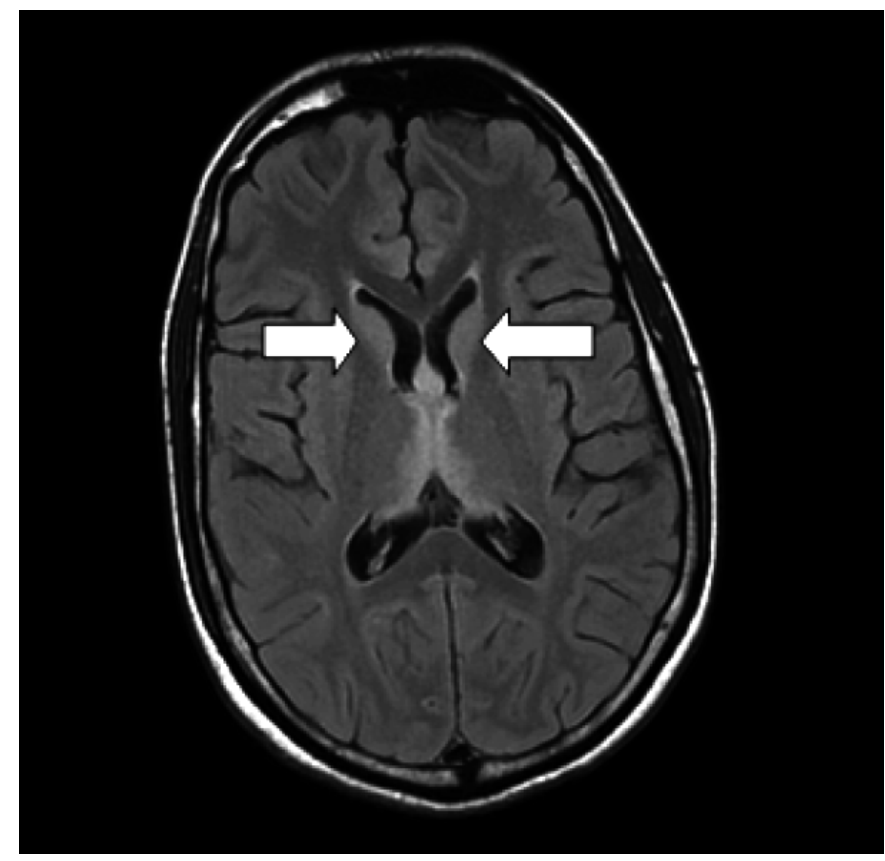

Fig. 3. MRI brain (axial) showing hyper-intensities in the caudate nucleus region demarcated by arrows.

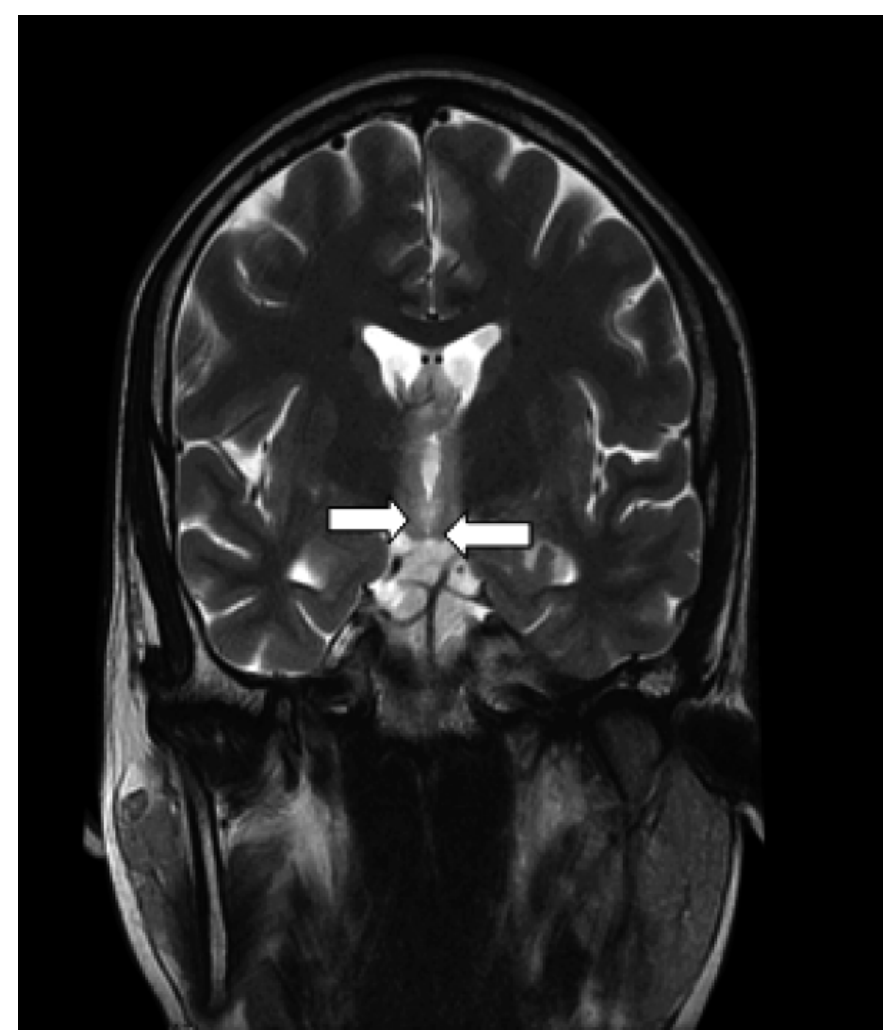

Fig. 4. MRI brain (axial) showing hyper-intensities in the mammillary bodies demarcated by arrows. evaluation returned positive for hepatitis A virus, which went a long way in explaining the protracted period of vomiting and jaundice.

During the course of his illness, our patient also developed confabulation, suggestive of the onset of Korsakoff's syndrome. However, with symptomatic treatment and thiamine supplementation, he improved gradually with a marked improvement in sensorium, ocular manifestations, and gait disturbance, which justified our provisional diagnosis of WE. But, disorientation and anterograde amnesia persisted. It was presumed that the protracted period of vomiting due to underlying hepatitis infection may have led to severe thiamine deficiency, which in turn may have precipitated the episode of WE. The patient was managed symptomatically for hepatitis A with thiamine supplementation. At the time of discharge, the patient was symptomatically better with mild residual disorientation and anterograde amnesia.

\section{Discussion}

WE, as defined in its classical sense, pertains to the clinical triad of mental confusion, ophthalmoplegia, and gait ataxia developed in the background of alcoholism. The diagnosis in such a scenario is straightforward. However, when the issue of gross under-diagnosis using the triad came to light, new criteria were proposed in which either 2 or more of the following features were mandated for the diagnosis of WE [9].

(1) Dietary deficiency,

(2) Oculomotor abnormalities,

(3) Cerebellar dysfunction,

(4) Altered mental status or memory impairment.

However, these criteria were not intended for the diagnosis in non-alcoholic patients, as in our patient's case, in which the index of suspicion is inherently low. A report involving various studies, case reports, and case series of WE until 2009 revealed that the clinical presentation among non-alcoholics was quite different from alcoholics [10]. The classical triad, as seen in our patient, was observed in only about a third of non-alcoholic WE patients. This number jumped up to about $56 \%$ among alcoholic patients [10]. A patient with no history of alcohol consumption and suffering from WE is more likely to present with non-specific symptomatology including nausea, vomiting along with features of underlying diseases which might precipitate thiamine deficiency, for instance, peptic ulcer diseases, pancreatitis, starvation or fasting, gastrointestinal surgery, bariatric surgery, AIDS, chemotherapy, and ageing. Furthermore, ocular manifestations and mental clouding were commonly observed among non-alcoholics than cerebellar symptoms [10]. This was further supported by the results of another systematic re- 
view involving Index Medicus and Medline databases, published in 2015 [11]. As per this systematic review, altered mental status was also the most common presentation. Besides the presenting features, the authors of the 2015 systematic review have also observed that due to any precipitating illness, WE develops significantly earlier than in case of alcohol disorder-induced WE. This observation is concordant with the scenario witnessed in our patient. The duration from the onset of hepatitis A till the development of WE was approximately 1 month. Thus, WE can develop in any individual, even in previously well-nourished individuals, who are bereft of a thiamine source for more than a few weeks and possess any of the underlying conditions mentioned here and more. Furthermore, in light of the varied and often non-specific presentation, the suspicion of WE should always be at the back of our minds in the presence of any one of the classic signs and symptoms.

Diagnosing WE, especially in a non-alcoholic patient, is further confounded by the absence of a reliable laboratory diagnostic method. Measuring erythrocyte thiamine transketolase activity level came closest to helping establish a diagnosis of thiamine deficiency. Presently though, it has been replaced by the measurement of serum thiamine levels $[12,13]$. However, these tests are not mandatory for the patient's management. Furthermore, the level of serum thiamine may not reflect the true thiamine levels in the brain. As in this case, once the diagnosis of WE is ascertained on the basis of clinical presentation, thiamine supplementation takes priority over laboratory evaluation [10].

Besides assessing the patient's thiamine levels, evaluation of the underlying cause for thiamine deficiency bears significance as unless the underlying pathology is taken care of, WE could progressively worsen and spill over into the realms of Korsakoff's psychosis. This is commonly observed in alcoholic patients [11]. However, our patient, despite being a non-alcoholic progressed to the stage of Korsakoff s psychosis. This was due to his underlying pathology of hepatitis A infection that led to severe thiamine deficiency, which remained undiagnosed for the 1 month period prior to hospitalisation and during the initial stages of hospitalisation.

In addition to laboratory parameters, imaging studies such as CT or MRI can be carried out to substantiate the diagnosis of WE. Their absence should not be the reason for the delayed treatment of the patient [10]. MRI has been found to be more sensitive than CT, more so in case of non-alcoholic patients. Furthermore, the sites of the lesions have been observed to be more atypical among

WE - 'Pushing the Envelope' of Patient's Profile non-alcoholic patients [10]. The typical sites of MRI lesions, as observed among alcoholic WE patients, include the thalamus, hypothalamus, mammillary bodies, and periaqueductal midbrain [10]. The atypical sites of the lesions in WE patients include cerebellum, vermis, cranial nerve nuclei, red nuclei, dentate nuclei, caudate nuclei, splenium, and cerebral cortex [10]. Besides, a close scrutiny of the size and the resolution of the lesions can be utilised in a prognostic capacity.

Despite it being a well-documented fact that thiamine supplementation helps correct thiamine deficiency, consensus is lacking on the issues related to the optimal dose, frequency of dose, duration of treatment [10]. Surprisingly, it has been observed that a dose of 100-200 mg intravenous thiamine is sufficient to reverse the disease process among non-alcoholics [10]. However, the dose required by alcoholics is much higher, and is in the range of $500 \mathrm{mg}$. The rationale behind this is yet to be established. It has been hypothesised that in case of alcoholics, previous sub-clinical episodes or the excess glutamate release by virtue of the co-existent alcohol withdrawal syndrome may lead to more severe brain damage, which in turn may warrant a higher thiamine supplementation [14].

Pharmacokinetic studies have indicated that with the half-life of thiamine being $96 \mathrm{~min}$ [15], a thrice daily dosing regimen may aid in sufficient concentration of thiamine reaching the brain than the single dose regimen [16]. In addition, catabolic conditions among non-alcoholic WE patients and alcohol consumption among alcoholic patients renders them unable to store thiamine. This is brought about by the down regulation of the enzymes associated with thiamine and the alteration of protein binding property induced by alcohol [10]. In such a situation, the intake of a balanced diet plays a significant role in the normalisation of the patient's overall condition, as observed in this case. Furthermore, thiamine supplementation should precede any carbohydrate intake as carbohydrate intake would end up utilising the miniscule amount of thiamine left in the body and worsen the WE further [17].

In light of repeated administration of thiamine for correction of WE, questions may arise over the safety of thiamine. Various studies have indicated that thiamine, irrespective of the route of administration, is a relatively safe drug with a few cases of anaphylaxis, generalised pruritus, and transient local irritation [18]. Even in our patient, despite administration of thiamine for a few weeks, there were no adverse reactions observed that could be attributed to thiamine administration. Thus, 
keeping in mind the available scientific evidence, our suggestions for the management of patients with WE without any history of alcohol consumption include:

(1) WE should be considered as a differential diagnosis whenever a non-alcoholic patient presents with ataxia, mental clouding, or ocular manifestations.

(2) A suspicion of WE is enough to warrant thiamine supplementation before glucose administration, irrespective of the availability of laboratory or imaging investigations.

(3) A meticulous history-taking is critical to uncover the underlying cause of WE. This can go a long way in the early treatment of WE and in mitigating the whole disease process at its initial stages.

(4) In the absence of well-established scientific evidence that substantiates the utility of laboratory tests in WE, at best EKTA levels or serum thiamine levels can be used in an adjunct capacity along with imaging studies. Treatment should not be delayed due to lack of laboratory or imaging results as the slightest delay can lead to irreversible brain damage.

(5) Administration of $500 \mathrm{mg}$ thiamine intravenously in a thrice daily regimen seems to be the norm. However, similar efficacy has also been observed with 100-200 $\mathrm{mg}$ among non-alcoholics. The standard practice followed is intravenous therapy for 5 days followed by an oral therapy, unless oral absorption of drugs is compromised.

(6) Treatment should be continued until the elimination of the pre-disposing risk factors for WE. The place of thiamine prophylaxis for healthy people is still controversial, and scientific evidence has been found to be lacking in this regard. ground of patients presenting with WE. The standard norms for the management of WE have been devised keeping in mind the patients with a history of alcoholism. However, in light of the increasing incidence among nonalcoholic patients, in whom the index of suspicion is inherently low due to non-specific symptomatology, it calls upon the physicians' clinical acumen to diagnose such cases. But, the morbidity and mortality associated with $\mathrm{WE}$ and its sequelae warrants that the diagnosis of WE among non-alcoholics be not left to the arbitrary capabilities of the clinician and that further research and con-

trolled studies be carried out to lay down more established guidelines with improved diagnostic sensitivity and better therapeutic outcomes.

\section{Acknowledgements}

The authors would like to acknowledge the support of Kasturba Hospital, Kasturba Medical College, Manipal and Manipal University in preparing this case report.

\section{Ethical Approval}

A written informed consent was obtained from the patient stating that the information pertaining to his case will be published without his name being attached to it. Furthermore, every attempt will be made to ensure anonymity. The Information may be published in a journal which could be read worldwide or may be placed on a website.

\section{Authorship Contribution}

B. Jayaprakash: acquisition and interpretation of data, approval of final version to be published; K.N. Rao: acquisition and interpretation of data; N. Patil and N.R. Rau: revision of the draft's intellectual content; D. Bhattacharjee: drafting and revision of the intellectual content; M. Maden: acquisition of data.

\section{Disclosure Statement}

The authors declare no conflict of interest. The authors declare that the manuscript complies with the Indian Council of Medical Journal Editors requirements.

\section{Conclusion}

This case is illustrative of the diverse medical back-

\section{References}

1 Wernicke C: Lehrbuch der Gehirnkrankheiten fur Aerzte und Studirende, ed 1. Berlin, Kassel Fischer, 1881.

2 Victor M, Adams RA, Collins GH: The Wernicke-Korsakoff Syndrome and Related Disorders Due to Alcoholism and Malnutrition. Philadelphia, F.A. Davis Company, 1989.

3 Martin PR, Singleton CK, Hiller-Sturmhöfel $\mathrm{S}$ : The role of thiamine deficiency in alcoholic brain disease. Alcohol Res Health 2003;27: 134-142.

4 Infante MT, Fancellu R, Murialdo A, Murialdo A, Barletta L, Castellan L, Serrati C: Challenges in diagnosis and treatment of Wernicke encephalopathy: report of 2 cases. Nutr Clin Pract 2016;31:186-190.

5 Zara G, Codemo V, Palmieri A, et al: Neurological complications in hyperemesis gravidarum. Neurol Sci 2012;33:133-135.
Jayaprakash/Rao/Patil/Bhattacharjee/ Maden/Rau 
6 Flabeau O, Foubert-Samier A, Meissner W, Tison F: Hearing and seeing: unusual early signs of Wernicke encephalopathy. Neurology 2008;71:694.

7 Davies SB, Joshua FF, Zagami AS: Wernicke's encephalopathy in a non-alcoholic patient with a normal blood thiamine level. Med J Aust 2011;194:483-484.

8 Bohan PK, Yonge J, Connelly C, Watson JJ, Friedman E, Fielding G: Wernicke encephalopathy after restrictive bariatric surgery. Am Surg 2016;82:73-75.

9 Caine D, Halliday GM, Kril JJ, Harper CG: Operational criteria for the classification of chronic alcoholics: identification of Wernicke's encephalopathy. J Neurol Neurosurg Psychiatry 1997;62:51-60.
10 Galvin R, Bråthen G, Ivashynka A, Hillbom $\mathrm{M}$, Tanasescu R, Leone MA: EFNS guidelines for diagnosis, therapy and prevention of Wernicke encephalopathy. Eur J Neurol 2010;17: 1408-1418.

11 Scalzo SJ, Bowden SC, Ambrose ML, Whelan G, Cook MJ: Wernicke-Korsakoff syndrome not related to alcohol use: a systematic review. J Neurol Neurosurg Psychiatry 2015;86: 1362-1368.

12 Tallaksen CM, Bøhmer T, Bell H, Karlsen J: Concomitant determination of thiamin and its phosphate esters in human blood and serum by high-performance liquid chromatography. J Chromatogr 1991;564:127-136.

$13 \mathrm{Lu}$ J, Frank EL: Rapid HPLC measurement of thiamine and its phosphate esters in whole blood. Clin Chem 2008;54:901-906.

14 Thomson AD, Marshall EJ: The natural history and pathophysiology of Wernicke's encephalopathy and Korsakoff's psychosis. Alcohol Alcohol 2006;41:151-158.
15 Tallaksen CM, Sande A, Bøhmer T, Bell H Karlsen J: Kinetics of thiamin and thiamin phosphate esters in human blood, plasma and urine after $50 \mathrm{mg}$ intravenously or orally. Eur J Clin Pharmacol 1993;44:73-78.

16 Donnino MW, Vega J, Miller J, Walsh M: Myths and misconceptions of Wernicke's encephalopathy: what every emergency physician should know. Ann Emerg Med 2007;50: 715-721.

17 Sechi G, Serra A: Wernicke's encephalopathy: new clinical settings and recent advances in diagnosis and management. Lancet Neurol 2007;6:442-455.

18 Wrenn KD, Murphy F, Slovis CM: A toxicity study of parenteral thiamine hydrochloride. Ann Emerg Med 1989;18:867-870. 